\title{
TENDENCIAS Y MODELOS EN LA EDUCACIÓN SUPERIOR EN CIRUGÍA “REINGENIERÍA EN EDUCACIÓN QUIRÚRGICA"
}

\author{
TENDENCIES AND MODELS IN SUPERIOR SURGICAL EDUCATION \\ "REENGINEERING IN SUGICAL EDUCATION"
}

\author{
William Sánchez Maldonado MD.Cirujano Oncólogo, FACS ${ }^{1 *}$ \\ ${ }^{1}$ Jefe del Servicio de Cirugía, Hospital Militar Central, Bogotá, Colombia. Docente Facultad de Medicina, \\ Universidad Militar Nueva Granada, Bogotá, Colombia
}

\begin{abstract}
"En la aplicación de un modelo de desarrollo educativo, si sistemáticamente y en forma continua no se logra el objetivo planeado, se debe considerar seriamente en hacer cambios profundos en la forma de actuar, que está cotidiana y profundamente arraigada".
\end{abstract}

La anterior frase originada en mi propia crítica del modelo de educación quirúrgica en Colombia, me lleva de una manera automática a pensar que en forma indiscutible se debe analizar y replantear un proceso de reingeniería educativa a este nivel, para poder responder a los retos contemporáneos de las disciplinas médicas quirúrgicas.

El modelo de educación médica y especialmente la quirúrgica que seguimos como base de la formación profesional, fue desarrollado por William Halsted en Baltimore, USA a finales del siglo XIX y principios del $\mathrm{XX}$. Este modelo que respondía a las necesidades contemporáneas del momento, introdujo la modalidad del internado y la residencia hospitalaria como pilares de la formación médica, para que el estudiante estuviera inmerso en un mundo de experiencia progresiva que le permitiera adquirir destrezas, habilidades y sobretodo, responsabilidades acumulativas para que al final de su entrenamiento lograra un estado de excelencia y así poder ejercer una práctica médica independiente, con el objetivo fundamental de ayudar a un paciente enfermo (1). Digo ayudar porque en muchas ocasiones no es posible curar a un paciente, pero siempre es factible ayudarlo. Este modelo de educación ha sido muy fructífero, con el que se han logrando grandes avances y en los cuales se fundamenta la práctica de la medicina moderna. Pero los tiempos cambian y las técnicas educativas deben evolucionar para responder a las necesidades sociales inmediatas.

Hoy es insostenible un modelo de educación en cirugía basado exclusivamente en la actitud pasiva y receptiva del estudiante, en donde no se cuestiona la enseñanza asistencial del profesor basada en la experiencia y experticia personal. La pregunta, la investigación científica y el debate deben ser el motor que impulsa la formación educativa de avanzada.

"Errar es humano ..." este aforismo frecuentemente referenciado en la práctica médica es tal vez el principal punto de reflexión que ha llamado a la evolución de la educación quirúrgica. El sociólogo Charles Bosk en su libro "Forgive and Remember: Managing Medical Failure" (Olvidar y Recordar: manejando el error médico), hace referencia a que el error normativo (moral) es punitivo, mientras el error técnico o el error en el juicio es frecuentemente aceptado y olvidado por el médico, contribuyendo a generaciones de cirujanos erráticos en el conocimiento, lo cual es desfavorable para el paciente (2). Hay quienes creen que existe el error honesto, y que el buen médico aprende de sus propios errores y el de los colegas. La esencia de la reflexión filosófica puede ser aceptada; sin embargo, hoy en día la humanidad ya no es contemplativa ni permisiva con el error en la práctica médica y los sistemas de control de calidad son veedores y controladores del acto médico. Es por esto que la educación quirúrgica debe evolucionar, la residencia en cirugía no debe desaparecer, y solo debe responder a las necesidades actuales de este mundo, que es diferente al de William Halsted.

* Correspondencia: William Sánchez SanchezWilliam@etb.net.co. Dirección postal: Tr.3 No 49-00, Servicio de Cirugía, Hospital Militar Nueva Granada, Bogotá, Colombia. 
Hoy día es indiscutible ejercer la medicina sin estar basado en la mejor evidencia, y entendiendo su aplicación dentro de un marco contextualizado de la realidad de su entorno práctico. Creo que si un cirujano no opera con el absoluto juicio de la razón (en toda su dimensión) y con todo el conocimiento existente, la realidad para el paciente se puede ir diluyendo y el riesgo puede emerger e incluso llegar a niveles no permitidos. "Aun la más elegante de las técnicas quirúrgicas realizada con un juicio o indicación errada, es inadmisible y posiblemente catastrófica".

En relación con el programa de formación en el área quirúrgica, clásicamente se ha basado en el estudio de libros y textos tradicionales y en la experiencia adquirida en las salas de cirugía y otros ambientes hospitalarios (urgencias, hospitalización, etc). En los últimos años este modelo ha sido cuestionado, y hoy día está en un proceso transición, pero aun no se ha encontrado el perfeccionamiento del modelo anhelado. El papel del docente ha evolucionado de un poseedor dictatorial del saber cercano a los dioses, a convertirse en un guía estimulador del ejerció mental y promotor del discernimiento y de la búsqueda de la verdad que debe encontrar el estudiante (3-7).

En Estados Unidos, según el Accreditation Council for Graduate Medical Education, la educación moderna debe estar basada en la búsqueda y en el desarrollo de las siguientes competencias: cuidado del paciente, conocimiento médico, profesionalismo, habilidades de comunicación interpersonal y social, práctica basada en la educación continuada y conocimiento de los sistemas de salud (4). En un mundo globalizado $y$ en especial en el que está la medicina, y en donde en todas las latitudes de nuestro planeta se exigen los resultados del tratamiento de una enfermedad y se comparan con el dato estadístico más exitoso, hace que sea necesario contemporizar las estrategias educativas para responder a las necesidades actuales de la profesión.

Debido a la crisis social y especialmente a la transgresión de los principios humanísticos fundamentales que se han visto desplazados y acorralados por el tecnicismo, es imperioso rescatarlos y retomarlos para que sean los pilares primordiales durante la formación y el desarrollo profesional, aun más en la medicina por su propia concepción.
El educador debe ser guía y garante del estudiante para que durante el desarrollo de las competencias establecidas en el plan de estudios institucional, construya un conocimiento eficiente y contemporáneo (útil para la sociedad y para sus propósitos) y además encuentre su propia razón de ser. En consecuencia, describiré bajo mi perspectiva y en forma muy global, que se debe hacer en cada una de las seis competencias actualmente establecidas en la educación médica:

1. Cuidado del paciente. La prioridad siempre es la seguridad del paciente y la búsqueda del mejor tratamiento. La educación está dirigida a adquirir destrezas médicas y quirúrgicas específicas, pero la sociedad e inclusive ya la ley, exigen que el aprendizaje se realice en modelos de simulación; una vez adquirida la experticia y habilidades necesarias, se ejecutan en el paciente y de esa forma hay una mayor probabilidad de omitir errores y de facilitar la búsqueda de un resultado terapéutico satisfactorio. El laboratorio de práctica es indispensable en cualquier programa de entrenamiento quirúrgico; los modelos de simulación son necesarios pero no reemplazan la práctica clínica, son complementarios. Actualmente no es aceptado el modelo de ensayo-práctica-error-práctica en el paciente.

2. Conocimiento médico. Se debe desarrollar un conocimiento expedito de las enfermedades, fundamentado en la literatura más relevante. El educador y el estudiante deben estar familiarizados con las ayudas tecnológicas modernas que facilitan encontrar permanentemente el conocimiento de manera inmediata, pero el estudiante debe capacitarse para diferenciar la información relevante de la no relevante. "El conocimiento es globalizado" por lo tanto hoy no se puede estudiar sin consultar en internet, sin buscadores, ni bases de datos computarizadas. El educador debe estimular el desarrollo de un conocimiento crítico en el estudiante, y permitir una apertura total a la controversia, debido a que esta es una parte esencial del motor del progreso científico. En la universidad, tanto el docente por su razón de ser como el estudiante por su propia condición, deben promover y desarrollar la investigación. La pregunta debe estar a la orden del día y su productividad debe ser documentada para las generaciones venideras que serán beneficiarias y testigo histórico de una de las mejores 
expresiones de la universidad. No es permisible en un medio universitario, que un docente y mucho menos un estudiante de avanzada, trascurran por el tiempo sin tener curiosidad científica, sin hacerse un cuestionamiento, sin plantearse preguntas. $\mathrm{Si}$ esto ocurre seguramente están fuera de contexto y en el lugar equivocado.

3. Profesionalismo. Este es un punto vital en la formación de un médico; actualmente se puede considerar que está en crisis en nuestro medio. El educador debe ser ejemplo y guía de la ética médica y la moral. En todas las profesiones y más aún en la medicina, se debe ser ante todo un buen ciudadano, con principios fundamentales en alto; en el proceso de formación se debe promover $y$ cultivar la honestidad, el respeto, la compostura, la ecuanimidad, la confidencia y la perseverancia; también entre otras virtudes y principios humanísticos está el asumir la responsabilidad ante el error.

\section{Habilidades de comunicación interper-} sonal y social. La medicina es una profesión interdependiente de la comunicación. Un gran porcentaje de los errores en la práctica médica están promocionados por la mala comunicación entre el personal involucrado en el caso o con el paciente. Con frecuencia las malas relaciones interpersonales, que generalmente son originadas por problemas de comunicación, convierten la vida cotidiana en algunos ambientes hospitalarios, en pruebas de supervivencia por la hostilidad generada en los trabajadores. También es muy importante realizar entrenamientos en modelos de atención de pacientes y familiares con estilo de vida y personalidades diferentes o difíciles (la definición de personalidad difícil es compleja, pero todos los días nos enfrentamos a ellas); igualmente es muy útil hacer dinámicas de grupo intrahospitalarias para una mejor convivencia y comunicación. A este respecto creo que deben ser muchas las sorpresas y muy valiosas las medidas para una mejor interrelación hospitalaria y profesional.

\section{Práctica basada en la educación continuada.}

La medicina y en especial la cirugía es un área del saber en constante y vertiginoso cambio. Un buen profesional médico, que pretenda estar activo, jamás podrá renunciar a la actualización del conocimiento. En el mundo y en especial en nuestro país, el debate sobre la interdependencia existente entre la industria farmacéutica, la industria de insumos quirúrgicos y la educación médica continuada en los posgrados, es un punto candente $\mathrm{y}$ de actualidad. Para nadie es un secreto que las sociedades científicas médicas, que son las que llevan el peso y son los garantes de la educación continuada, dependen económicamente de los recursos provenientes de la industria. Esto, unido a la baja remuneración salarial que deja en el ultimo escaño de la escala de prioridades de un médico padre de familia, la renovación de conocimiento, y finalmente, la falta de políticas claras del Estado en esta materia son unas de las principales y fundamentales causas de los complejos problemas de la mala práctica médica cotidiana en nuestro país (por supuesto no generalizada). Para garantizar el nivel de excelencia médica que merecen recibir los colombianos es necesario adoptar políticas de estado, que garanticen la educación médica continuada para todos los profesionales.

6. Conocimiento y práctica de los sistemas de salud. Todo programa educativo en medicina y cirugía debe contemplar la formación y preparación para los diferentes escenarios de la práctica del futuro profesional. El educador debe preparar al estudiante para laborar tanto en la práctica privada como en la estatal, a nivel urbano o a nivel rural. Más aun, se debe contemplar en forma obligada escenarios internacionales por la indudable globalización en materia de salud. A este respecto es muy preocupante la situación de crisis del sector salud en Colombia, que fomenta el desprofesionalismo y que está llevando a la ausencia de profesionales altamente calificados (en todo su sentido y significado de la palabra) en las especialidades quirúrgicas. Por ejemplo, medicina dejó de ser la depositaria de los mejores cocientes intelectuales determinados por los exámenes de estado; ya no es una profesión tan apetecida por los bachilleres y tampoco lo son sus especialidades quirúrgicas, principalmente por el alto esfuerzo físico, emocional y económico para llegar a ser un especialista que no compensa con los beneficios a percibir.

Desde el punto de vista económico, para formar un supraespecialista en cirugía (tórax, transplante, oncología, vascular, etc) se requiere de trece a quince años de estudio posteriores a la educación básica, periodo en el cual no recibe remuneración 
alguna y se tiene que pagar matrículas semestrales y conseguir dinero para su subsistencia básica (¿dónde o cómo lo puede conseguir, si la educación le exige dedicación completa y exclusiva?). Si el estudiante no tiene contratiempos finalizará su formación entre los 35-40 años de edad, con una deuda acumulada que podrá pagar en un tiempo no inferior a doce años, si se tiene en cuanta la remuneración percibida en el mercado laboral actual. ¿Cómo alguien puede pretender estudiar hoy en día una especialidad quirúrgica con esas perspectivas laborales? Hoy ya estamos viendo la respuesta a este raciocinio, que se manifiesta en un menor número de candidatos en los programas de subespecialidades. Pero ¿qué será de los pacientes del futuro? Ante esta perspectiva, el gobierno nacional debe reaccionar especialmente, en lo relacionado con la educación médica, que esta en poder del estado y de sus sabias decisiones futuras.

En conclusión, la educación médica y en especial la quirúrgica, está actualmente en transformación; es un proceso complejo que tiene varios actores, entre ellos, las universidades, el Estado, los docentes y los estudiantes. Las universidades, que en su autonomía legalmente establecida por la ley, deben afrontar con mucha responsabilidad, el reto social e histórico dejando de lado el hoy muy visible interés monetario manifiesto en muchas instituciones de carácter privado. El estado, que en su ejercicio y legislación, debe crear los mecanismos de control que realmente garanticen la calidad en educación, pero sobre todo ser corres- pondiente con las urgentes e inmediatas necesidades en materia de salud que tenemos los colombianos. A este respecto es preocupante las medidas tomadas en esta materia en el decreto 2376 del 1 de julio del 2010 (8), que deja a la educación médica con un futuro muy sombrío. Y por supuesto los docentes y estudiantes desempeñando sus roles respectivos con experticia y excelencia para garantizar un ejercicio justo, correcto y eficiente de su profesión. "El tiempo pasara y veremos que ocurrió".

\section{Referencias}

1. Thorwald Jürgen. El siglo de los cirujanos. Ediciones destino $5^{\mathrm{a}}$ edición. Barcelona.2000.

2. Bosk Charles. Forgive and Remember: Managing Medical Failure. 2 nd ed. Chicago. University Chicago. 2003.

3. O'Connor E.S, et al. Developing a practice-based learning and improvement curriculum for an academic general surgery resident. J Am Coll Surg. Vol 210,N4, April 2010.

4. ACGMME. Outcome Project. www.acgme.org/outcome/comp/ compCPRL.asp.

5. Canal DF, et al. Practice-based learning and improvement: a curriculum in continuous quality improvement for surgery residents. Arch Surg. 142;479-482.2007.

6. Siri J, et al. A multidisciplinary systems based practice learning experience and its impact on surgical residency education. $J$ Surg Edu.64;328-332.2007.

7. Larkin A.C, et al. Human emotion and response in surgery (HEARS) A simulation-based curriculum for communication skills, systems-based practice, and professionalism in surgical residency training. J Am Coll Surg. Vol 211,N²;285-292. August 2010.

8. Ministerio de la Protección Social, Colombia. Decreto 2376 del 1 de Julio del 2010. Por el que se regula la relación docenciaservicio para el programa de formación de talento humano en salud. 